\title{
EL CÁLCULO DE LA LENTE INTRAOCULAR TRAS CIRUGÍA FOTO-REFRACTIVA CORNEAL. REVISIÓN DE LA LITERATURA
}

\section{INTRAOCULAR LENS POWER CALCULATION AFTER CORNEAL PHOTOREFRACTIVE SURGERY; LITERATURE REVIEW}

\author{
MESA-GUTIÉRREZ JC ${ }^{1}$, RUIZ-LAPUENTE C ${ }^{2}$
}

\begin{abstract}
RESUMEN
Objetivo: Revisión de las distintas fórmulas de corrección del poder refractivo de la córnea (Kpost) y de la estimación de la posición efectiva de la lente (ELP) para la cirugía de cataratas después de cirugía refractiva corneal.

Método: Revisión de la literatura médica.

Resultados: Es necesario realizar una corrección de la Kpost para el cálculo de la lente intraocular tras cirugía refractiva. Existen diferentes vías dependiendo de la información disponible. Para estimar la ELP es necesario realizar una nueva corrección. Es aconsejable disponer de la queratometría previa (Kpre) y posterior (Kpost) a la cirugía y la refracción previa (Rpre) y posterior (Rpost). De esta manera, podremos realizar la corrección doble$\mathrm{K}$ de Aramberri en la fórmula de cálculo.

Conclusiones: Es conveniente registrar la refracción y queratometría ( $\mathrm{K}$, y método) previamente a la cirugía refractiva: la Kpre es crítica para el cálculo de la ELP.
\end{abstract}

Palabras clave: Lente intraocular, cirugía refractiva, potencia dióptrica corneal, cirugía cataratas, corrección doble-K, posición efectiva de la lente.

\begin{abstract}
Objective: Revision of methods for estimating corneal power (Kpost) and effective lens position (ELP) for cataract surgery after corneal refractive surgery.

Methods: Review of medical literature.

Results: To calculate the intraocular lens that achieves emetropia after corneal refractive surgery it is necessary to correct the Kpost and to estimate the effective lens position again correcting it. There are different formulae depending on the available information. Ideally, we need pre (Kpre) and posttreatment keratometry record (Kpost) and pre (Rpre) and post (Rpost) refraction. In this way, we can use the Aramberri's double-K method in the formula for calculating intraocular lens power.

Conclusions: Previously to keratorefractive procedure is advisable to have pre-treatment refraction and keratometry ( $\mathrm{K}$ and method) registered: Kpre value is critical to calculate the ELP (Arch Soc Esp Oftalmol 2009; 84: 283-292).
\end{abstract}

Key words: Intraocular lens, refractive surgery, corneal refractive power, cataract surgery, doble-K correction, effective lens position.

\footnotetext{
Recibido: 7/8/08. Aceptado: 26/6/09.

Servicio de Oftalmología. Hospitales Universitarios Virgen del Rocío. Sevilla. España.

1 Doctor en Medicina, EBOD (European Board of Ophthalmology). Hospitales Universitarios Virgen del Rocío y Hospital Universitario de Bellvitge.

2 Doctor en Medicina. Hospital Universitario Virgen del Rocío.

Correspondencia:

Juan Carlos Mesa Gutiérrez

Cl. Argantonio $3,4 .^{\circ} \mathrm{D}$

41008 Sevilla

España

E-mail: jcarlosmesa@mixmail.com
} 


\section{INTRODUCCIÓN}

Cada vez es más frecuente programar para cirugía de cataratas a pacientes a los que previamente se ha efectuado cirugía refractiva. El problema que surge con tales pacientes es el de calcular una lente intraocular de potencia adecuada para conseguir un grado de ametropía satisfactorio.

La sorpresa refractiva tras la cirugía de cristalino es una situación frecuente, con una incidencia del 100\% si no se efectúan las correcciones oportunas (1). Las razones son dos: un cálculo incorrecto de la potencia corneal y una estimación incorrecta de la posición efectiva de la lente (effective lens position, ELP).

Tras cirugía refractiva corneal la utilización del valor queratométrico medido, sin efectuar ninguna corrección, producirá un cálculo erróneo de la potencia de la lente intraocular: un paciente tratado de miopía que se someta a cirugía de cataratas quedará hipermétrope y al contrario, si previamente se trató una hipermetropía, tras la cirugía de catarata quedará miope.

Si solamente efectuamos esta corrección nuevamente obtendremos una miopía o hipermetropía residual: la razón es una estimación incorrecta de la ELP si solamente utilizamos en la fórmula de cálculo el valor de la Kpost.

El propósito de esta revisión es proporcionar las herramientas necesarias para la corrección de la Kpost y así obtener un nuevo valor, la Kpost corregida (Kpost-corr) que es el que deberemos introducir en la fórmula de cálculo juntamente con el valor previo a la cirugía refractiva láser corneal (Kpre) para realizar una estimación adecuada de la ELP y obtener así un cálculo correcto de la potencia de la lente intraocular.

\section{SUJETOS, MATERIAL Y MÉTODOS}

La búsqueda, selección y cuantificación de resultados se realizó de por dos investigadores independientes (JCMG y CRL), con el siguiente consenso preestablecido.

\section{Estrategia de búsqueda}

Se acude a la base de datos Medline y a la red Internet. Para obtener datos de fiabilidad para el cálculo de la lente intraocular tras cirugía refractiva en Medline, entre enero de 2001 y diciembre de 2008, se utilizaron las opciones de escrutinio recomendadas por los expertos (2-4). Se tantearon diferentes palabras clave (Lente intraocular, cirugía refractiva, poder refractivo corneal, queratometría post-operatoria, corrección doble-k y posición efectiva de la lente), descriptores compuestos y metatérminos en orden creciente de especificidad hacia el tema estudiado, seleccionando y cruzando los más productivos y selectivos (2-4). Se leyeron los resúmenes de todos los trabajos publicados en inglés o castellano y se recopilaron todos los artículos completos incluidos en el estudio. La búsqueda se complementó con un vaciado en Internet (Firefox-Google) al efecto de encontrar publicaciones científicas fiables omitidas en Medline (4,5).

\section{Selección de artículos para el análisis}

\section{Criterios de inclusión}

- El objetivo fundamental del trabajo es valorar la fiabilidad en el cálculo de la lente intraocular para cirugía de cataratas en pacientes previamente intervenidos con cirugía corneal foto-refractiva.

- El estudio aporta datos necesarios de la historia clínica oftalmológica y detalla el problema ocular antes de la intervención.

- Los materiales y métodos permiten reproducir la investigación.

- Se especifica cuándo y cómo se observan los resultados, aportando pruebas objetivas.

- Se trata de una publicación reconocida por un organismo científico.

\section{Criterios de exclusión}

- Comunicaciones en congresos, experiencias de investigadores no publicadas, opiniones de autor, etc.

\section{Estadística descriptiva de agrupación}

Se considera el número de ojos intervenidos en cada estudio (n), y el grado de consecución de emetropía tras la cirugía de cataratas. Para valorar la fiabilidad del método de cálculo (FMC) en cada artículo se establece la siguiente escala cuantitativa: 
error refractivo $>-1$ dioptrías [-1], emetropía [0], error refractivo $>+1$ dioptrías [1]. Para puntuar cada trabajo se tuvieron en cuenta los resultados de la refracción postoperatoria.

Se anotó para cada trabajo la refracción conseguida tras la cirugía de cataratas. También los datos más destacados de la discusión y conclusión de cada trabajo.

Las puntuaciones de la FMC se pautaron previamente por ambos investigadores. Se define como fiabilidad del método de cálculo ponderado (FMCP) el producto FMC x n, y fiabilidad global, o grupal (FMCG), al cociente FMCP/FM, donde FM es la fiabilidad máxima.

\section{Validación de los resultados}

Para validar la fiabilidad interobservador en la búsqueda, selección de artículos y puntuación de fiabilidad en la escala cuantitativa se calculó el índice Kappa (K). Se consideró concordancia válida si $\mathrm{K}>0,7$. Las discrepancias entre ambos investigadores se anularon por consenso utilizando el razonamiento crítico-deductivo $(6,7)$.

Analizada toda esta información mostramos diversas vías para estimar la Kpost y proponemos la corrección en la fórmula de cálculo de la LIO según dispongamos o no de un dato clave: la queratometría previa a la cirugía refractiva.

\section{RESULTADOS}

Encontramos diferentes métodos para efectuar la corrección de la potencia dióptrica de la córnea tras la cirugía refractiva y así obtener el valor denominado Kpost-corregida (Kpost-corr). Básicamente, dichos métodos pueden clasificarse en aquellos que utilizan el cambio en equivalente esférico producido o aquellos que utilizan el valor proporcionado por la topografía corneal.

El cálculo de la lente intraocular (LIO) en pacientes intervenidos de cirugía refractiva láser corneal es mucho más complejo de lo normal, pues además de tener longitudes axiales extremas, que ya complica por sí mismo el cálculo, se añaden factores por la cirugía previa que alteran la predictibilidad de las fórmulas existentes $(8,9)$.

Por tanto, el proceso de cálculo de la potencia de la LIO debe modificarse cuando se practica en un ojo sometido a cirugía foto-refractiva corneal. Existen dos fuentes de error: la incorrecta predicción de la ELP por parte de la fórmula y la determinación errónea de la potencia de la córnea por parte de la queratometría (10). La corrección de estos dos factores permite realizar un cálculo correcto en estos ojos:

1. Predicción de la ELP (método doble-k): se debe utilizar la $\mathrm{K}$ previa a la cirugía corneal en el algoritmo de predicción de la ELP y la Kpost en el cálculo de vergencia como potencia de la primera lente del sistema.

2. Determinación de la potencia corneal tras cirugía refractiva: Tras la cirugía refractiva ablacional la superficie anterior de la córnea se aplana, sin que cambie la cara posterior. Esta relación alterada conduce a una sobrestimación de la potencia corneal por parte de los queratómetros: se hace necesario realizar una corrección de la Kpost.

Se han descrito varios métodos que permiten determinar correctamente la potencia de la córnea, dependiendo de los datos de los que dispongamos (1). En líneas generales, podemos encontrarnos ante tres posibles situaciones (fig. 1):

1. Conocemos la queratometría y refracción preoperatorias (Kpre y Rpre)

2. Sólo conocemos la Rpre, y la refracción y queratometría postoperatorias (Rpost y Kpost).

3. No conocemos ningún dato anterior a la cirugía.

Kpre será el valor medido antes de la cirugía refractiva corneal, si se dispone de él. En caso contrario puede calcularse sumando las dioptrías corregidas en córnea al valor que definamos como Kpost.

\section{Conocemos todos los datos: Kpre, Kpost, Rpre y Rpost}

\section{a) Método de la Historia Clínica (MHC) $(11,12)$}

Kpost-corr $=$ Kpre - EEpre + EEpost

EEpre: Equivalente esférico pre-operatorio.

EEpost: Equivalente esférico post-operatorio.

Pongamos como ejemplo un paciente con una Kpre $=49,25 D$, una Rpre (EEpre) en plano de gafas de -8 D y una Rpost=-1D.

EEpre (distancia vértex $12 \mathrm{~mm}$ ): -8 D

Rpre en plano corneal: $-8 /(1-[0,012 *-8 *])=$ 7,30 D. 


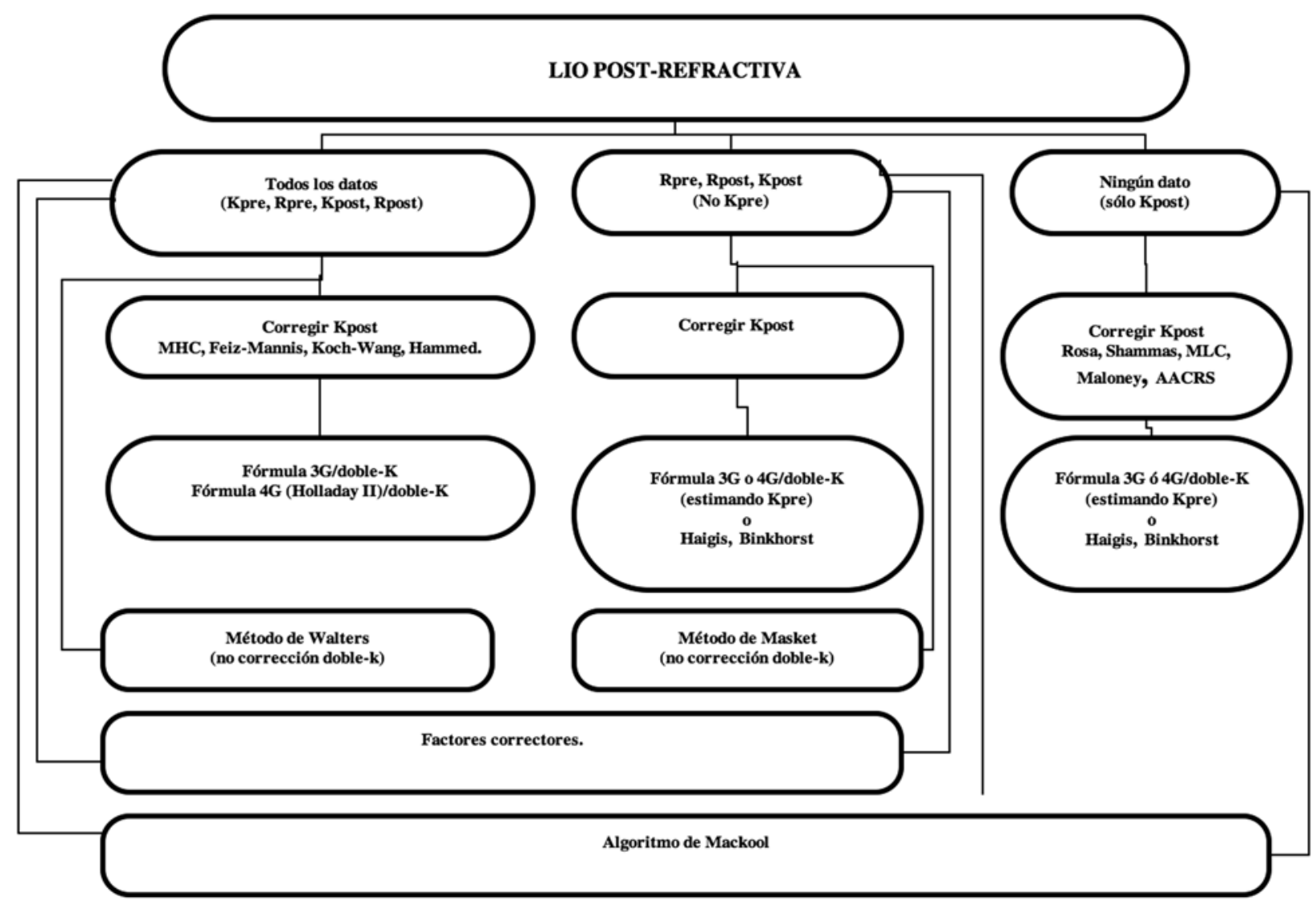

Fig. 1: Algoritmo cálculo de la LIO.

EEpost (distancia vértex $12 \mathrm{~mm}$ ): $-1 \mathrm{D}$

Rpost en plano corneal: $-1 /(1-[0,012 *-1])=-0,98$

Rpost-Rpre $=-7,30-(-0,98):-6,32 \mathrm{D}$

Kpost-corregida $=$ Kpre-corrección $=49,25-$ $6,32=42,93 \mathrm{D}$.

A continuación podemos utilizar la fórmula SRK/T con la corrección doble-K (Kpre y Kpostcorregida) de Aramberri (13).

\section{b) Modificación de K topoqueratométrica (14)}

Kpost-corr: Kmedia (SimK)-15\% dioptrías corregidas.

Ejemplo:

$\operatorname{simK}=37 \mathrm{D}$,

corrección de 10D,

Kpost-corr $=37-1,5=35,5 \mathrm{D}$.

Introducimos los datos en cualquier fórmula con corrección doble-K. c) Método de Feiz-Mannis (15)

Se calcula potencia de la LIO como si el paciente no se hubiera sometido a cirugía refractiva. Añadimos el cambio inducido por el LASIK en el error refractivo $(\Delta \mathrm{D})$ dividido entre 0,7 .

LIOpost $=$ LIOpre $+(\Delta \mathrm{D} / 0,7)$

\section{d) Método de Koch-Wang (16)}

Realizamos una topografía corneal y tomamos el valor EffRp (effective refractive power).

Kpost-corr $=\operatorname{EffRp}(\Delta \mathrm{D} \times 0,19)$

e) Método de Hammed $(17,18)$

Tomamos igualmente el valor EffRp y realizamos la correción:

Kpost-corr $=$ EffRPadj $=$ EffRp $-(\Delta \mathrm{D} / 0,15)$ 


\section{f) Índices topográficos del Orbscan y Pentacam (19)}

Los mapas que calculan la potencia paraxial son el Mean Total Power en el Orbscan y el Net Power en el Pentacam. Los topógrafos de hendidura escaneada permiten medir las caras anterior y posterior de la córnea, siendo posible obtener directamente la potencia total de la córnea sumando los valores reales de ambas superficies. Por tanto, podremos evitar la asunciones en que se basan los queratómetros y topógrafos de Plácido $(K=1,3375)$. Esta $K$ no es la auténtica potencia paraaxial de la córnea central, ya que el índice de refracción que mejor aproxima dicho valor es de 1,3315. Sin embargo, 1,3375 es el valor que utilizan las fórmulas de vergencia más empleadas. Por tanto, los valores obtenidos con el Orbscan y el Pentacam se convierten mediante la suma de un factor a un equivalente del índice queratométrico estándar $\mathrm{K}$ (1,3375): para el Pentacam Net Power sería +0.95 y $+1,1$ para el Orbscan Mean Total Power (MTP).

Ejemplo: ojo operado de 4 dioptrías de miopía.

SimK $=40,7$ dioptrías.

$\mathrm{MTP}=38,86$.

$38,86+1,1=39,86$

\section{g) Método del bypass corneal de Walter}

Introducimos la Kpre y la longitud axial (AXL) en la fórmula de cálculo con una refracción diana equivalente a la refracción pre-LASIK.

Ejemplo:

Refracción pre-LASIK $=-11,75$ con Kpre = 43,87 y $\mathrm{AXL}=28,54 \mathrm{~mm}$.

La refracción diana que introduciremos en la fórmula de cálculo será $-11,75$.

Con este método no necesitamos la Kpost ni su estimación, no siendo necesaria la corrección doble-K en la fórmula de cálculo (20).

\section{Sólo conocemos la Rpre, Rpost y Kpost}

\section{a) Diferencia de refracción (1)}

Nuevamente debemos estimar la Kpost. Continuando con el ejemplo, si la Rpre $=-8 \mathrm{D}$ y la Rpost $=-$ $1 \mathrm{D}$, la corrección en el plano corneal será

Rpost-Rpre $=-7,30-(-0,98):-6,32 \mathrm{D}$

Supongamos que la Kpost=39,25D. Utilizando la corrección propuesta por Feiz (11), obtenemos que:
Kpost-corregida=Kpost- $0,23 *$ corrección

Kpost-corregida=39,25-0,23*6,32

Kpost-corr $=37,79 \mathrm{D}$.

A continuación introducimos los datos en la fórmula de cálculo. Como no disponemos de la Kpre, no podremos realizar la corrección doble-K, por lo que deberíamos utilizar la fórmula de Holladay-II (sólo disponible previo pago como software comercial) o la de Haigis (21) o Binkhorst (que no utilizan los valores K).

\section{b) Utilización de factores correctores}

Es el método más sencillo, pero no exento de error. Podemos utilizar el nomograma de Feiz (tabla 1) (22), que nos calcula la potencia de la LIO según el cambio producido en EE o el nomograma de Koch (tablas II y III) que nos calcula dicha potencia según las dioptrías corregidas y la longitud axial (23).

c) Por supuesto, para corregir la Kpost también podemos utilizar los métodos descritos en el apartado anterior, pero al no disponer de la Kpre no podremos realizar la corrección doble-K.

Asumiendo determinados valores podríamos arriesgarnos a calcular una Kpre para poder realizar la corrección doble-K en la fórmula de cálculo. Por ejemplo, paciente que dice haber sido operado hace 10 años de unas 8 dioptrías con PRK, la Kpost es de

Tabla I. Nomograma de Feiz

\begin{tabular}{cc}
\hline Cambio en EE (plano gafa) & $\Delta$ Potencia LIO (D) \\
\hline 1 & 0,6 \\
1,50 & 0,66 \\
2 & 0,96 \\
2,50 & 1,26 \\
3 & 1,55 \\
3,50 & 1,85 \\
4 & 2,15 \\
4,50 & 2,45 \\
5 & 2,74 \\
5,50 & 3,04 \\
6 & 3,34 \\
6,50 & 3,64 \\
7 & 3,93 \\
7,50 & 4,23 \\
8 & 4,53 \\
8,50 & 4,83 \\
9 & 5,12 \\
9,50 & 5,42 \\
10 & 5,72 \\
10,50 & 6,02 \\
11 & 6,31 \\
11,50 & 6,61 \\
12 & 6,91 \\
\hline \hline
\end{tabular}


Tabla II. Nomograma para el cálculo de la LIO tras cirugía refractiva miópica. Cifra que ha de ser añadida a la potencia calculada utilizando SRK-/T, Hoffer Q y Holladay I

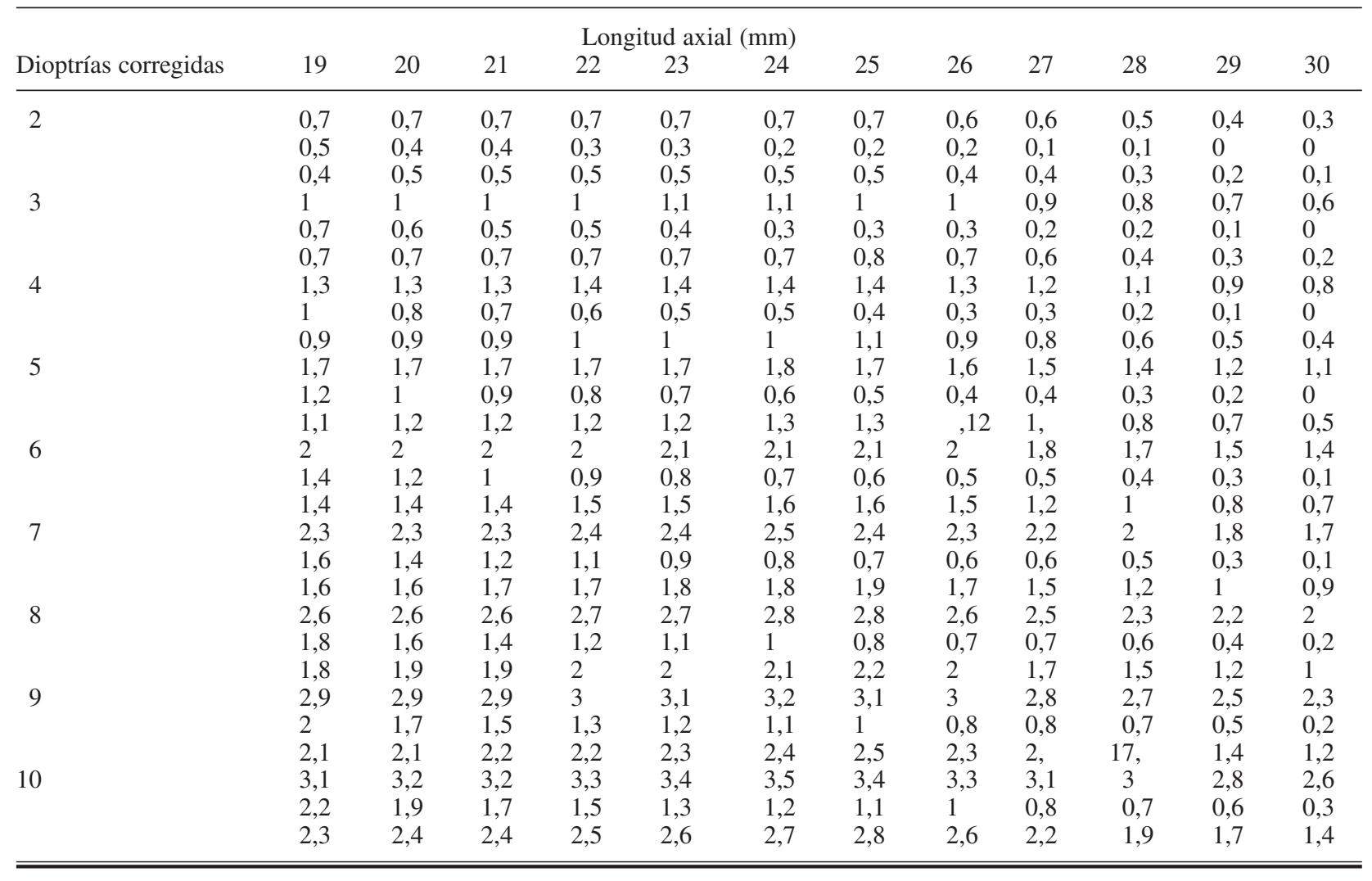

Tabla III. Nomograma para el cálculo de la LIO tras cirugía refractiva hipermetrópica. Cifra que ha de ser restada a la potencia calculada utilizando SRK-/T, Hoffer Q y Holladay I

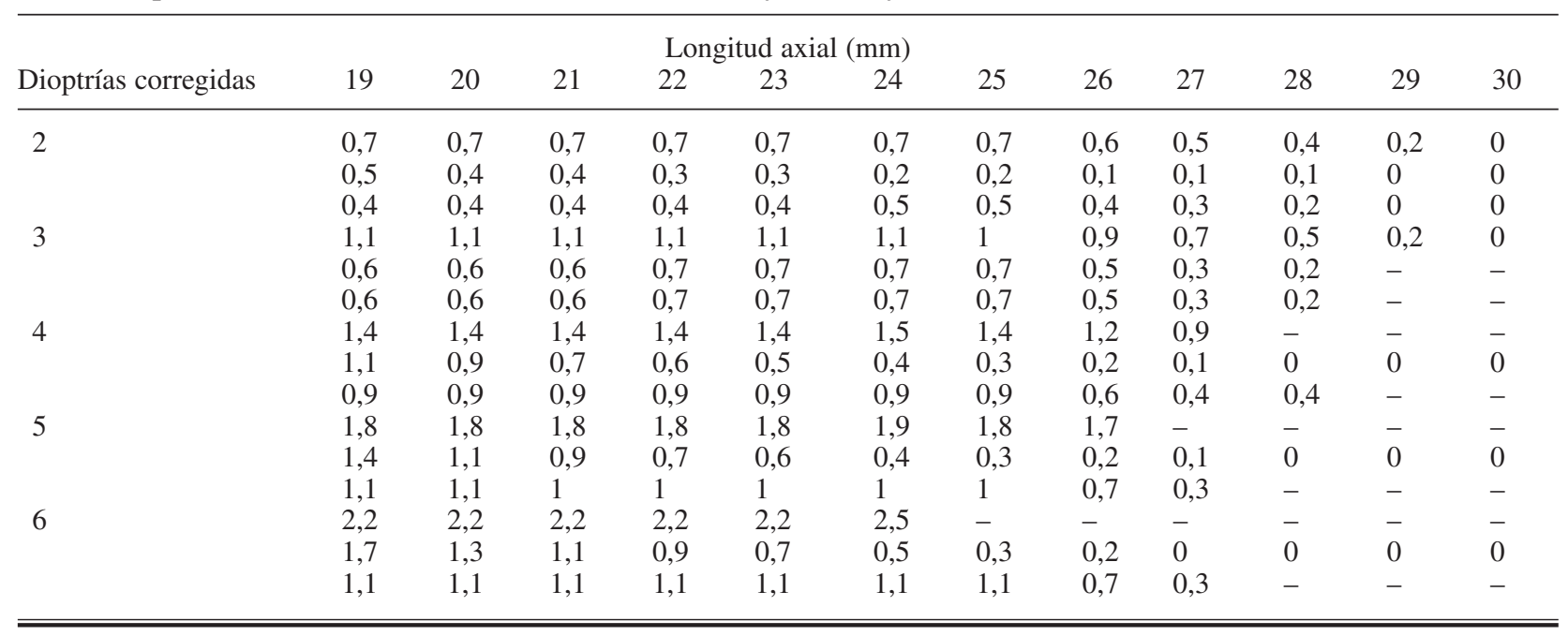

37. Asuminos una corrección en gafa de 8D, que se traduce en 7,30 en plano corneal. Calculamos Kpost restando $15 \%$ de 7,30 (es decir, 1,09 ) a 37:
Kpost $=37-1,09=35,91$.

Kpre podemos calcularla sumando $35,91+7,30$ $=43,21$. 
De esta forma introduciremos en la fórmula Kpre $=43,21$ y Kpost $=35,91$.

\section{d) Método de Masket}

Realiza un ajuste en la fórmula de regresión, añadiéndolo en el caso del LASIK miópico y restándolo en el LASIK hipermetrópico.

Ajuste de potencia de la LIO = EEpre $*(-0,326)$ $+0,101$

Ejemplo:

Con la fórmula SRK/T nos sale una LIO $=16$ para la emetropia.

EEpre $=-6$

Ajuste de potencia $=-6 *(-0,326)+0,101=+2,057$.

Añadimos 2 a la LIO originalmente calculada: 16 $+2=18 \mathrm{D}$.

Por tanto, implantaremos una LIO de +18 dioptrías (24).

No es necesaria la corrección doble-K con este método.

\section{No sabemos ningún dato anterior a la cirugía: sólo Kpost}

Medimos la K de forma habitual (en el ejemplo Kpost $=39,25)$. A continuación, corregimos el valor Kpost mediante dos fórmulas de regresión:

\section{a) Fórmula de Rosa (25)}

Refracción corregida con fórmula de Rosa (Rro$\mathrm{sa})=\mathrm{R}(0.0276 \mathrm{LA}+0,3635)$

LA=longitud axial; $\mathrm{R}=\mathrm{k} / 337,5$

Por tanto, la $\mathrm{K}$ postoperatoria estimada $[$ Kpost $($ Rrosa $)]=337,5 /$ Rrosa

Ejemplo: $\mathrm{LA}=25,5$

$\mathrm{R}=337,5 / 39,25=8,5987$

Rrosa $=8,5987 *(0,7038+0,3635)=9,17$

Por tanto, Kpost $($ Rosa $)=36,80$

La fórmula de Rosa utiliza sólo la fórmula SRK (SRK/T si LA<=29,4 mm o SRK-II si LA >29,4 mm).

\section{b) Fórmula de Shammas (26)}

Kpost(Shammas) $=1,14$ K-6,8.

Kpost $($ Shammas $)=1,14 * 44,25-6,8=50,45-6,8$
Kshammas $=43,65 \mathrm{D}$

El método de Shammas sólo utiliza la fórmula de Shammas.

\section{c) Método de la lente de contacto (27)}

Realizamos una refracción subjetiva. A continuación colocamos una lente de contacto rígida de PMMA de una curva base (potencia) conocida y se realiza una nueva refracción. Si la refracción no ha cambiado, la córnea tiene igual potencia que la lente de contacto. Si la refracción es más miópica, la lente de contacto es más curva (más potencia) que la córnea y lo contrario pasará en la hipermetropía. Estimamos la Kpost.

Rpost $=$ Refracción en gafa postoperatoria $=-1 \mathrm{D}$

$\mathrm{Rlc}=$ Corrección con la lente de contacto $=+1 \mathrm{D}$.

Curva base $=\mathrm{CB}=40$

DR (diferencia de refracción $)=$ Rlc-Rpost $=+1-(-$ $1)=+2$

Kpost-corr $=\mathrm{CB}+\mathrm{DR}=39,25+2=41,25 \mathrm{D}$.

\section{d) Método de Maloney (24)}

Necesitamos una topografía y estimamos la Kpost a partir de EffRp

Kpost-corr= (EffRp x 1,114)-6,1

e) Método de la Asociación Americana de cirugía refractiva y cataratas (19)

Este método permite calcular el radio posterior de la córnea en función de las dioptrías corregidas. El objetivo es cuantificar la razón cara anterior/cara posterior antes y después de la cirugía. El resultado es que esta razón es bastante constante en todo el rango de córneas no operadas (40,55-47,2):1,25 $( \pm 0,3)$ (media y desviación estándar). Tras la cirugía la razón se hace variable, con un incremento proporcional a las dioptrías corregidas, pudiendo ajustarse una relación lineal:

Razón Ant/Post=1,257 + 0,032 x dioptrías corregidas en córnea.

Mediante esta fórmula podemos calcular, a partir de un radio de curvatura anterior obtenido por topografía o queratometría, el radio de la cara posterior y a continuación la potencia total de la córnea. Si la Simk es de 37 D, obtenemos el radio anterior: 
n2-n1/dioptrías:

$0,3375 / 37=9,12 \mathrm{~mm}$

Si aplicamos la fórmula razón Ant/Post:

$$
1,257+0,032 \times 10=1,58
$$

Obtenemos rpost:

$9,12 / 1,58=5,77 \mathrm{~mm}$

Si lo convertimos a dioptrías:

$1,336-1,376 / 5,77=-6,93$

Calculamos la potencia de la cara anterior: $0,376 / 9,12=41,23$

Sumamos superficies y grosor corneal:

$$
41,23+0,1-6,93=34,4
$$

Esta es la potencia paraxial real de la córnea.

También podríamos estimar la Kpre según lo visto en el apartado 2, para sí intentar realizar la corrección doble-K.

Una medida de control interesante es emplear la fórmula de Haigis, que no emplea la $\mathrm{K}$ como predictora de la ELP, para validar el resultado. En esta fórmula únicamente introduciremos LA, profundidad de cámara anterior (ACD) y Kpost. Dicha fórmula no está publicada en una revista peer-review pero podemos obtener las constantes de la LIO en la página web de la Universidad de Wuerzburg (21).

\section{f) Algoritmo de Mackool}

Se realiza la cirugía de catarata y se deja al paciente afáquico. Aplicamos el algoritmo de Mackool:

Refracción en afaquia $(\mathrm{EE}) * 1,75=$ potencia de la LIO.

En una ventana de tiempo entre el mismo día de la cirugía y las 3 semanas realizamos el implante de la LIO (28).

\section{Sorpresa refractiva}

Si a pesar de realizar las correcciones adecuadas el resultado es un error refractivo podemos realizar un recambio de LIO según la tabla IV (29).

\section{DISCUSIÓN}

El cálculo inexacto de la potencia dióptrica de la LIO a implantar en la cirugía de catarata tras la realización de cirugía refractiva es un problema de importancia creciente.
Tabla IV. Correcciones para recambio de LIO

\begin{tabular}{lcc}
\hline $\begin{array}{l}\text { EE preoperatorio } \\
\text { (D) }\end{array}$ & $\begin{array}{c}\text { Hipermetropía residual: } \\
\text { aumento } \\
\text { de potencia } \\
\text { de la LIO (D) }\end{array}$ & $\begin{array}{c}\text { Miopía residual: } \\
\text { disminución de } \\
\text { potencia } \\
\text { de la LIO(D) }\end{array}$ \\
\hline 1 & 0,21 & 0,68 \\
1,50 & 1,04 & 1,26 \\
2 & 1,87 & 1,84 \\
2,50 & 2,70 & 2,42 \\
3 & 3,53 & 3,00 \\
3,50 & 4,36 & 3,58 \\
4 & 5,19 & 4,16 \\
4,50 & 6,02 & 4,74 \\
5 & 6,85 & 5,32 \\
5,50 & 7,68 & 5,90 \\
6 & 8,51 & 6,48 \\
\hline \hline
\end{tabular}

Nota: Valores absolutos de EE.

La alteración en la relación entre la cara anterior y posterior de la córnea tras un procedimiento fotorefractivo y la utilización del índice queratométrico estándar condiciona que la lectura queratométrica aportada por los queratómetros o por los topógrafos sea inexacta, y condiciona un error en el cálculo de la ELP y de la potencia de la lente intraocular $(1,10)$.

La potencia dióptrica total de la córnea es la suma de la potencia de la cara anterior (lente convexa) y de la posterior (lente cóncava). Tras la cirugía foto-refractiva se produce un cambio en la curvatura de la superficie anterior (aplanándose en al caso de la cirugía foto-refractiva miópica e incurvándose tras la cirugía por hipermetropía), mientras que no cambia la superficie posterior.

La queratometría tradicional y la queratometría simulada por la topografía corneal estima la potencia corneal midiendo los 3,2 $\mathrm{mm}$ centrales de la superficie anterior. Para una cornea normal prolata esta asunción es adecuada, pero tras cirugía refractiva la relación se altera. Los instrumentos que miden tanto la superficie anterior como la posterior, como el Orbscan y el Pentacam pueden disminuir este error en la determinación de la potencia corneal total.

La alteración del índice de refracción tras cirugía refractiva corneal es otra fuente de error: los queratómetros estándar se basan en un índice de refracción de la córnea de 1,3375 para convertir el radio de curvatura a potencia dióptrica y obtener una potencia media corneal promediando las potencias dióptricas de la superficie corneal anterior y poste- 
rior. Tras la cirugía refractiva la relación entre los radios de curvatura anterior y posterior de la córnea ya no es de 7,5/6,3, lo que origina un error en el cálculo de la ELP $(9,24,25)$. Así, tras cirugía fororefractiva son inválidos los valores de los distintos índices de refracción corneales (índice de refracción estándar=1,3375; SRK/T=1,3333) que sí permiten el cálculo del poder refractivo total de la córnea a partir del radio de curvatura de la superficie anterior de la córnea en ojos no intervenidos $(1,14,23,24)$.

Por tanto, medir la potencia corneal neta no es lo más indicado ya que los valores utilizados por los queratómetros y topógrafos no se cumplen en estos ojos. Un problema añadido es que no podemos cuantificar la desviación entre el cambio en la potencia refractiva de la córnea medido y el cambio refractivo para determinar un factor de corrección. Aunque se han propuesto valores medios (14-25\% del cambio refractivo), la dispersión es demasiado alta, probablemente como resultado de los cambios en la curvatura corneal posterior $(10,14,24,25)$.

Además, la sorpresa refractiva postoperatoria puede ser explicada por la ineficacia de la fórmula de cálculo cuando utilizamos solamente la Kpost. Las fórmulas de tercera generación (SRK/T, Hoffer/Q, Holladay-I, Haigis) calculan la posición de la lente respecto de la córnea (la ELP) midiendo la profundidad de cámara anterior (en el caso de la fórmula de Haigis) o estimando dicha profundidad por medio de la queratometría (SRK/T, Hoffer/Q, Holladay-I). Si la queratometría no es exacta, trasladamos dicho error al cálculo de la ELP. Cualquier fórmula teórica de $3{ }^{a}$ generación realiza dos pasos: primero, utiliza la AXL y la K para calcular la profundidad de cámara anterior o anterior chamber depth (ACD). Después, ésta variable, junto con la AXL y la K nuevamente, se utilizan para calcular la potencia dióptrica de la LIO. Si consideramos que en el primer paso se realiza una estimación de la profundidad de la cámara anterior (ACD) y que esta distancia anatómica no cambia después de la cirugía refractiva, parece obvio que utilizar un valor de $\mathrm{K}$ menor que el original (el que resulta de la cirugía refractiva) nos proporcionará una infravaloración de la ELP y por tanto de la potencia de la LIO, con la consiguiente sorpresa refractiva postoperatoria $(10,14,24,25)$.

Aramberri propone una modificación en la fórmula SRK/T en la cual la Kpre se utiliza para estimar la ELP y la Kpost para calcular el poder dióp- trico de la LIO (fórmula doble-K). Cualquier fórmula de 3. ${ }^{a}$ generación puede corregirse para que utilice la Kpre para el cálculo de ELP y la Kpost para el cálculo de la potencia de la LIO (13).

$\mathrm{Si}$ disponemos de todos los datos (queratometría y refracción pre y post-operatorias) el método de cálculo que parece más fiable es el de la historia clínica $(1,9)$. Si no disponemos de la Kpre, podemos utilizar los nomogramas de Koch (16). En el peor de los casos, cuando sólo disponemos de la Kpost, solamente nos queda la posibilidad de realizar al corrección de ésta mediante el método de Rosa, que parece ser bastante fiable, o los métodos de Shammas y de la lente de contacto, un tanto más complicados estos últimos en tanto que el método de Shammas utiliza una fórmula propia y para el MLC se requiere un juego de lentes especiales (23-25). Lógicamente, al no disponer de Kpre no podremos reaizar la corrección doble-K siendo esperable un error residual de entre 1-3 dioptrías (14,20-34).

Una importante conclusión sería que registrar la Kpre es crítico para el cálculo de la ELP. De hecho, sólo en aquellos pacientes en los que dispongamos de la historia refractiva previa (Kpre y Rpre) podremos estimar la ELP utilizando una fórmula de 3 . $^{\mathrm{a}}$ generación con la corrección doble-K de Aramberri $(10,13)$.

\section{BIBLIOGRAFÍA}

1. Mesa JC, Martí T, Arruga J. Cálculo de la potencia de la lente intraocular en situaciones especiales. Annals d'Oftalmologia 2008; 16: 68-89.

2. Dickersin K, Scherer $R$, Lefevre C. Identifying relevant studies for systematic reviews. BMJ 1994; 309: 1286-1291.

3. Helmer D, Savoie I, Green C, Kazanjian A. Evidencebased practice: extending the search to find material for the systematic review. Bull Med Libr Assoc 2001; 89: 346352.

4. Soualmia LF, Dahamna B, Thirion B, Darmoni SJ. Strategies for health information retrieval. Stud Health Technol Inform 2006; 124: 595-600.

5. Darmoni SJ, Thirion B, Leroyt JP, Douyère $M$, Lacoste B, Godard C, et al. A search tool based on «encapsulated» $\mathrm{MeSH}$ thesaurus to retrieve quality health resources on the internet. Med Inform Internet Med 2001; 26: 165-178.

6. Shahar E. A Popperian perspective of the term «evidencebased medicine». J Eval Clin Pract 1997; 3: 109-116.

7. Osborn J. Observation, Sherlock Holmes, and Evidence Based Medicine. Med Secoli 2002; 14: 515-527.

8. Iribarne Y, Ortega Usobiaga J, Sedó S, Fossas M, Martínez Lehmann P, Vendrell C. Cálculo del poder dióptrico de lentes intraoculares. Annals d'Oftalmologia 2003; 11: 152-165. 
9. Mesa JC, Martí T, Arruga J. Cálculo del poder dióptrico de la lente intraocular (LIO) tras cirugía refractiva. Arch Soc Esp Oftalmol 2005; 80: 699-704.

10. Borasio E, Stevens E, Smith GT. Estimation of true corneal power after keratorefractive surgery in eyes requiring cataract surgery: BESSt formula. J Cataract Refract Surg 2006; 32: 2004-2014.

11. Speicher L. Intra-ocular lens calculation status after corneal refractive surgery. Curr Opin Ophthalmol 2001; 12: 17-29.

12. Seitz B, Langenbucher A. Intraocular lens calculation status after corneal refractive surgery. Curr Opin Ophthalmol 2000; 11: 35-46.

13. Aramberri J. Intraocular lens power calculation after corneal refractive surgery: double-K method. J Cataract Refract Surg 2003; 29: 2063-2068.

14. Gimbel H, Sun R, Kaye GB. Refractive error in cataract surgery after previous refractive surgery. J Cataract Refract Surg 2000; 26: 142-144.

15. Gimbel H, Sun R, Furlong MT. Accuracy and predictability of intraocular lens power calculation after laser in situ keratomielusis. J Cataract Refract Surg 2001; 27: 571576.

16. Koch D, Wang L. Calculating IOL power in eyes that have had refractive surgery. J Cataract Refract Surg 2003; 29: 2039-2042.

17. Hamed A, Wang L, Misra M, Koch C. A comparative analyisis of five methods of determining corneal refractive power in eyes that have undergone myopic laser in situ keratomileusis. Ophthalmology 2002; 109: 651-658.

18. Feiz V, Mannis M, Garcia-Ferrer F, Kandavel G, Darlington J, Kim E. Intraocular lens power calculation after laser in situ keratomieleusis for myopia and hyperopia: a standardized approach. Cornea 2001; 20: 792-797.

19. Aramberri J. Cálculo de la lente intraocular tras cirugía refractiva corneal. In: Alió J, Rodríguez-Prats J. Buscando la excelencia en la cirugía de la catarata. Barcelona: Editorial Glosa; 2006; 179: 191.

20. Walter K, Gagnon M, Hoopes P, Dickinson P. Accurate intraocular lens power calculation alter lase in situ keratomieleusis, bypassing corneal power. J Cataract Refract Surg 2006; 32: 425-429.

21. Haigis W. IOL calculation according to Haigis. Disponible en: http://www.augenklinik.uni-wuerzburg.de/uslab/ ioltxt/haie.htm

22. Feiz V, Moshirfar M, Mannis M, Reilly C, Garcia-Ferrer F. Nomogram-based intraocular lens power adjustment after myopic photokeratectomy and Lasik. Ophthalmology 2005; 112: 1381-1387.

23. Wang L, Booth M, Koch D. Comparison of intraocular lens power calculation methods in eyes that have undergone LASIK. Ophthalmology 2004; 111: 1825-1831.

24. Masket S, Masket SE. Simple regresión formula for intraocular lens power adjustment in eyes requiring cataract surgery after excimer laser photoablation. J Cataract Refract Surg 2006; 32: 430-434.

25. Rosa N, Capaso L, Romano A. A new method of calculating intraocular lens power alter photorefractive keratectomy. J Cataract Refract Surg 2002; 18: 720-724.

26. Shammas H, Shamas M, Garabet A, Kim J, Shammas A, LaBree L. Correcting the corneal power measurements for intraocular lens power calculations after myopic laser in situ keratomileusis. Am J Ophthalmol 2003; 136: 426-432.

27. Haigis W. Corneal power after refractive surgery for myopia: contact lens method. J Cataract Refract Surg 2003; 29: 1397-1411.

28. Mackool RJ, Wilson K, Mackool R. Intraocular lens power calculation after laser in situ keratomieleusis: aphakic refraction technique. J Cataract Refract Surg 2006; 32: 435-437.

29. Jin W, Crandall A, Jones J. Intraocular lens exchange due to incorrect lens power. Ophthalmology 2007; 114: 417424.

30. Savini G, Barboni P, Zanini M. Intraocular lens power calculation alter myopic refractive surgery: theoretical comparison of different methods. Ophthalmology 2006; 113: 1271-1282.

31. Fam HB, Lim KL. A comparative análisis of intraocular lens power calculation methods alter myopic laser surgery. J Cataract Refract Surg 2008; 24: 355-360.

32. Srivannaboon $S$, Rapkanichmanee T, Cheng A, Fam $H$. Estimation of posterior corneal power for IOL calculation alter myopic LASIK. J Cataract Refract Surg 2008; 24: 946-951.

33. Awwad S, Manasseh C, Bowman R, Cavanagh H, Verita $S$, Mota V. Intraocular lens power calculation after myopic laser in situ keratomieleusis: Estimating the corneal refractive power. J Cataract Refract Surg 2008; 34: 10701076.

34. Khalil M, Choksshi A, Latkany R, Speaker MG, Yu G. Prospective evaluation of intraocular lens calculation after myopic refractive surgery. J Cataract Refract Surg 2008; 24: 33-38. 